\title{
Simultaneous imaging of neural activity in three dimensions
}

\author{
Sean Quirin*, Jesse Jackson, Darcy S. Peterka * and Rafael Yuste
}

Department of Biological Sciences, Columbia University, New York, NY, USA

Edited by:

Edward M. Callaway, The Salk

Institute for Biological Studies, USA

Reviewed by:

Hollis Cline, The Scripps Research

Institute, USA

Timothy E. Holy, Washington

University, USA

Bernardo Sabatini, Howard Hughes

Medical Institute, USA

*Correspondence:

Sean Quirin and Darcy S. Peterka,

Department of Biological Sciences,

Columbia University, 901 NWC

Building, 550 West 120th Street,

PO Box 4822, New York, NY 10027,

USA

e-mail: seanquirin@gmail.com;

dp2403@columbia.edu
We introduce a scanless optical method to image neuronal activity in three dimensions simultaneously. Using a spatial light modulator and a custom-designed phase mask, we illuminate and collect light simultaneously from different focal planes and perform calcium imaging of neuronal activity in vitro and in vivo. This method, combining structured illumination with volume projection imaging, could be used as a technological platform for brain activity mapping.

Keywords: three-dimensional imaging, calcium imaging, volume imaging, spatial-light-modulator, brain activity map

\section{INTRODUCTION}

Optical imaging of neural activity has several advantages over alternative strategies such as patch electrodes or electrode arrays. First, it is minimally invasive and allows for the monitoring of large ensembles of neurons with single-cell resolution (Yuste and Katz, 1991). In addition, it is compatible with a large variety of functional sensors (voltage, calcium or metabolic indicators, either exogenous or genetically encoded), and can be used for chronic imaging of defined cell populations in vitro or in vivo (Grynkiewicz et al., 1985; Kralj et al., 2012; Chen et al., 2013).

However, some key weaknesses remain for functional imaging of neuronal cell activity. From the very first microscope designed by Leeuwenhoek, image acquisition is typically limited to a single plane, while nearly all biological structures are threedimensional, thus requiring sequential scanning for volumetric imaging. As a result of this sequential scanning, the sampling rate over the volume is slow relative to neuronal activity $(1-100 \mathrm{~ms})$. Moreover, in highly scattering tissue, although two photon excitation has afforded single cell resolution and imaging below superficial layers (Horton et al., 2013), images are still mostly generated by serially scanning a single beam (for exceptions see Gobel et al., 2007; Cheng et al., 2011; Katona et al., 2012). Temporal focusing techniques can deliver high-fidelity illumination patterns into scattering tissue within a limited field-of-view but still require axial scanning with a moving objective, potentially coupling mechanical motion into the sample (Schrödel et al., 2013). Despite recent advances to increase the image acquisition speed, few proposals are scalable toward meeting the ultimate goal of fast, population voltage imaging with single cell resolution in scattering tissue - a task that would require millisecond temporal resolution across wide spatial areas (Alivisatos et al., 2012). Here we present an alternative to traditional scanning-based imaging by taking advantage of programmable three-dimensional illumination with spatial light modulators (SLM) to simultaneously excite neurons located at different focal points, together with a wavefront coded imaging approach that enables us to collect light from all focal points simultaneously. Our technique therefore supersedes scanning by illuminating and collects light from the neurons of interest in parallel.

\section{MATERIALS AND METHODS WAVEFRONT CODING FOR VOLUME PROJECTION MICROSCOPY}

While SLM based multi-site excitation has been coupled to widefield microscopy before, it has been limited to $2 \mathrm{D}$ or planar acquisition (Nikolenko et al., 2008; Anselmi et al., 2011). In contrast, we use a phase-only SLM to create multiple well-defined beamlets of light that optically sample many neurons throughout the sample volume. The wavefront coded imaging system then creates a $2 \mathrm{D}$ projection of this extended sample volume onto the detector plane. Our wavefront coded imaging system is physically realized by a phase mask placed between an image relay of the microscope pupil (Figure 1A) and is optimized for highspeed, three-dimensional data acquisition in what we call Volume Projection Imaging (VPI) (Dowski and Cathey, 1995; Quirin et al., 2013). Cells are no longer restricted to a single plane and can be freely distributed throughout the three-dimensional sample (Figure 1B). We note that although in some samples (transparent or effectively sparse), single photon excitation is sufficient, we regard two-photon capabilities as essential in turbid environments and for allowing precise modulation of activity when coupled with optogenetics or caged compounds (Papagiakoumou et al., 2010; Packer et al., 2012).

Operationally, in our method, high spatial-resolution structural volume data is first acquired via conventional single beam 


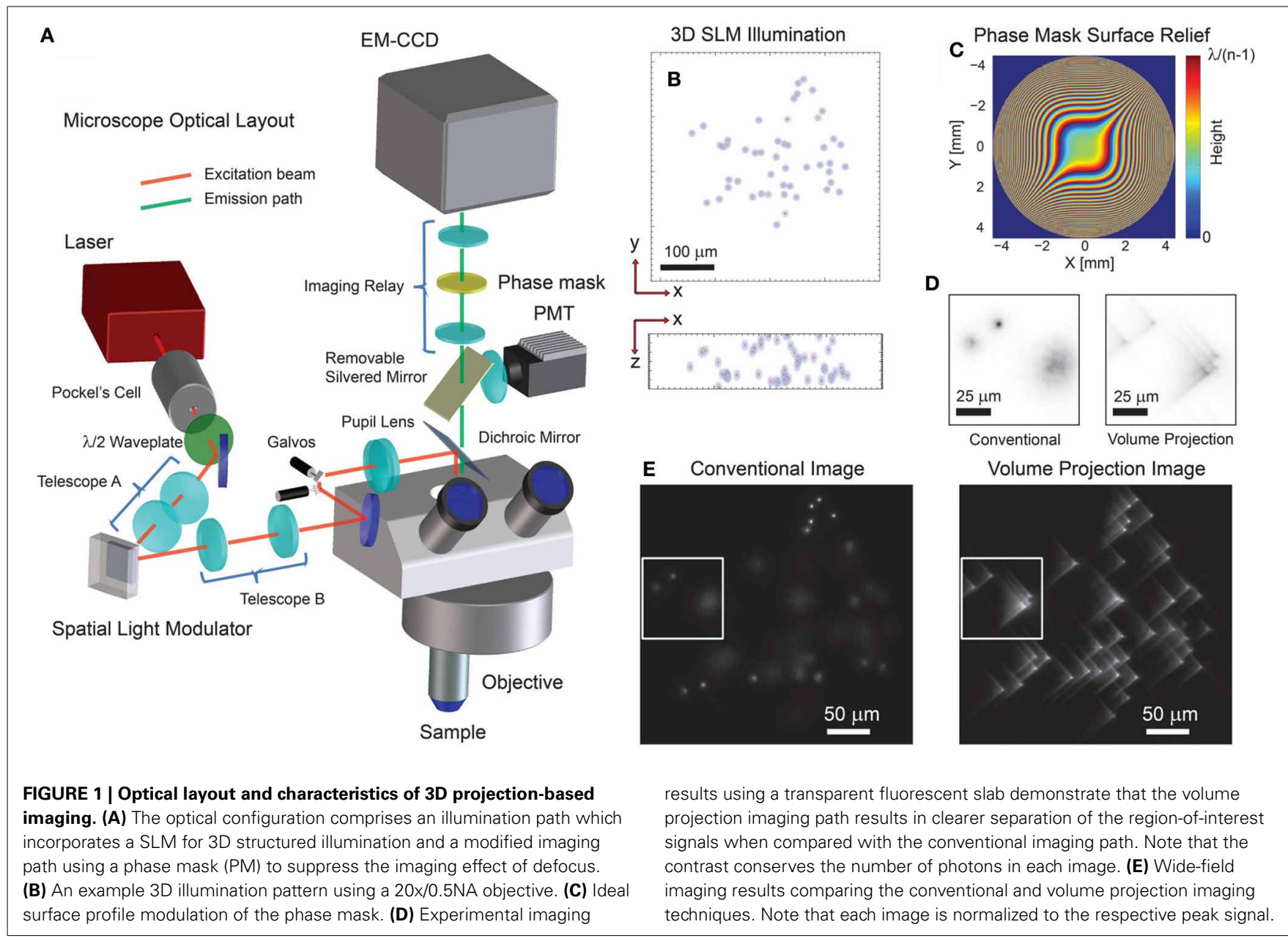

two-photon raster scanning. This volume data is then processed to identify the cells to be targeted by the SLM. Using these targets, we generate a hologram (phase pattern) that when displayed on the SLM creates the 3D structured illumination which illuminates the regions of interest (ROIs) in the volume (see Quirin et al., 2013 and references therein). Data is acquired by imaging the fluorescent activity of all of the ROIs simultaneously by the cubic phase wavefront coded imaging system regardless of their locations in 3D space (Cathey and Dowski, 2002; Quirin et al., 2013). The deterministic illumination provided by the SLM provides a priori knowledge which can be used to extract additional information from the collected image, as explained below.

For simultaneous 3D signal acquisition, our wavefront coded imaging system increases the standard limited depth-of-field, while maintaining the full aperture, and hence collection efficiency, of the objective. This custom imaging system uses a phase-only optical mask in the imaging path to effectively null defocus effects in the image (Dowski and Cathey, 1995). The effect of this is to greatly reduce the axial dependence of the point spread function (PSF) (i.e., the image is invariant to 3D position), which allows us to record an aberrated line-of-sight projection of the sample volume onto the image plane. This phase mask is described by the complex amplitude profile,

$$
p(x, y)=\left\{\begin{array}{cc}
e^{i 2 \pi \alpha\left(x^{3}+y^{3}\right)} & \text { if } x^{2}+y^{2} \leq 1 \\
0 & \text { otherwise }
\end{array}\right.
$$

where we used values of $\alpha=17$ and 12 for $M=20 \mathrm{x} / 0.5 \mathrm{NA}$ and $40 \mathrm{x} / 0.8 \mathrm{NA}$ objectives, respectively, and $x, y$ are the normalized coordinates of the microscope pupil (Figure 1C).

The trade-off for this defocus invariance is a decreased contrast in the focused image (Figure 1D) (Cathey and Dowski, 2002; Quirin et al., 2013). As a result, the acquired wide-field image exhibits a characteristic blur (e.g., an aberrated Point Spread Function) which is now essentially invariant regardless of the three-dimensional location of the ROI (Figure 1E). This blur is nonetheless spatially restricted, and reduction of the out-offocus PSF size compared with traditional imaging allows for a much higher local spatial density of ROIs (Figure 1D). Now, fluorescence signals from throughout the volume can be simultaneously acquired without loss of contrast due to out-of-focus collection (Figure 1E). As an additional benefit, the data volume is projected onto a $2 \mathrm{D}$ image plane thus reducing the total data throughput required during acquisition. In principle, this image can be digitally restored to diffraction-limited resolution by use of deconvolution methods-however, these steps are not 
necessary here. Instead we report results using a least-squares optimal signal extraction method described in section Targeting Calibration, Image Acquisition and Signal Reconstruction, which had not been applied in earlier methods.

\section{MICROSCOPE SETUP}

The structured-illumination microscope with VPI uses a single laser for exciting fluorescence of either the calcium indicator dye (Fura2-AM) or a genetically-encoded calcium indicator (GCaMP5G). A detailed optical layout of the system is given in Figure 2. The laser source is a Coherent Mira HP $(\sim 140 \mathrm{fs}$ pulses, $80 \mathrm{MHz}$, linear polarization) which can be manually tuned between $\sim 720$ and $1100 \mathrm{~nm}$. At $\lambda=800 \mathrm{~nm}$, the system provides $3.8 \mathrm{~W}$ and at $\lambda=920 \mathrm{~nm}, 2.2 \mathrm{~W}$ (for Fura2-AM and GCaMP5G excitation, respectively). The output beam was directed through an electro-optic (EO) modulation device (Pockels cell) to modulate intensity on the sample (ConOptics EO350-160). A broadband $\lambda / 2$ waveplate (Thorlabs AHWP05M-980) was located after the EO modulator to rotate the polarization state to be parallel with the active axis of the Spatial Light Modulator (Boulder Nonlinear Systems, XY-512) located further downstream. A shutter (Vincent Associates, LS6Z2) was placed in the beam path in order to control illumination state condition (i.e., "ON" and "OFF"). A 1:2 telescope ( $11=50 \mathrm{~mm}, \mathrm{f} 2=100 \mathrm{~mm}$, Thorlabs plano-convex lenses) scales the optical beam to approximately $8 \mathrm{~mm}$ to fill the active area of the SLM. The expanded beam is then redirected to the SLM by a periscope. The SLM has a custom look-up table which was experimentally determined at both wavelengths reported for use. The angle of incidence of the illumination beam to the SLM was $\sim 10^{\circ}$. A $4: 1$ telescope ( $\mathrm{f} 3=300 \mathrm{~mm}, \mathrm{f} 4=75 \mathrm{~mm}$, Thorlabs plano-convex lenses) reduces the image of the SLM onto a set of galvanometer mirrors (Cambridge $6210 \mathrm{H}, 4 \mathrm{~mm}$ open aperture). The galvanometer mirrors are located conjugate to the microscope objective pupil of an Olympus BX-51 microscope by use of an Olympus pupil transfer lens $(\mathrm{f} 5=50 \mathrm{~mm})$ and the mounted tube lens ( $\mathrm{fTL}=$ $180 \mathrm{~mm}$ ). Two microscope objectives were used for the experiments presented in the manuscript-an Olympus $40 \mathrm{x} / 0.8 \mathrm{NA}$ WI (slice experiments) and an Olympus 20x/0.5NA WI objective (in vivo experiments), though many other objectives performed similarly during informal testing. In practice, due to transmission losses from the passive and active optical elements, such as the EO modulator and the SLM (from pixel-cross talk, pixel fill factor, etc.), and from beam vignetting at the telescope after the SLM, the overall transmission efficiency from laser to sample was approximately $30 \%$.

When operating in conventional two-photon raster scanning mode, Olympus Fluoview was used to control the galvanometers and digitize the signal from a Photo-Multiplier Tube (PMTHamamatsu H7422P-40) located above the microscope. During PMT imaging a removable silvered mirror (M3, mounted in Thorlabs LC6W and LB4C) redirect the collected fluorescence emission into the PMT module.

To operate in volume projection mode, custom software was developed under MATLAB (The MathWorks, Natick, MA) which loads the correct look-up table and generates a hologram for the SLM that targets the selected ROIs located throughout the sample volume. When the illumination shutter is opened, the sample is illuminated with a custom light pattern that addresses the targeted cell somata. For VPI acquisition, the PMT redirection mirror, M3, is removed and the fluorescence emission is passed through an optical relay system with the custom phase mask. The

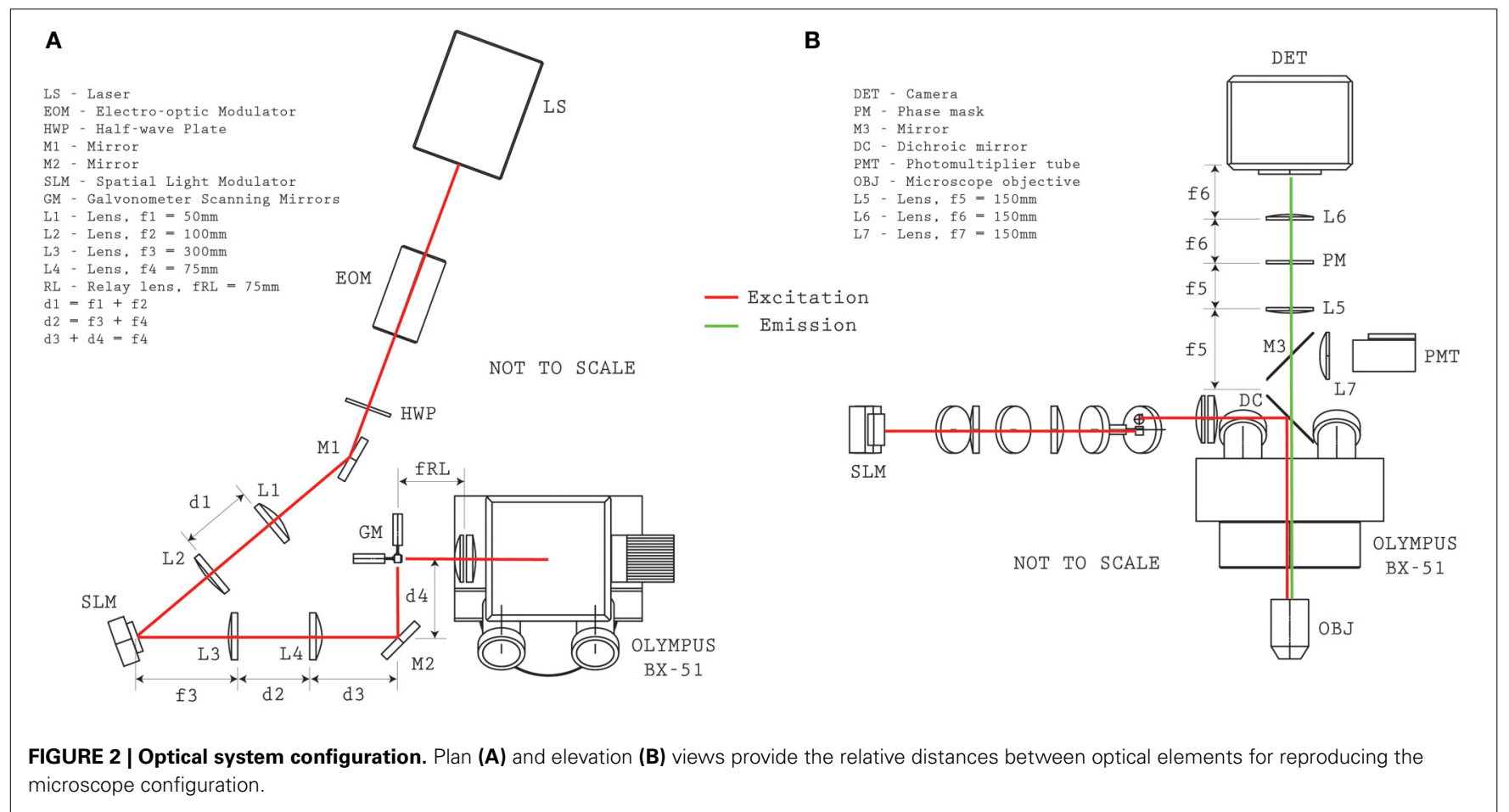


optical relay consists of a 1:1 telescope $\left(\mathrm{f} 4 \mathrm{f}=150 \mathrm{~mm}\right.$, Thorlabs $2^{\prime \prime}$ achromatic doublets) with a custom designed surface relief patterned onto a $1^{\prime \prime}$ diameter quartz plate that is placed a distance $\mathrm{f} 4 \mathrm{f}$ behind the first lens. Note that no amplitude modulation is necessary (i.e., the mask is transparent), critical for high sensitivity imaging, and that the surface relief of the mask is described by,

$$
h(x, y)=\left(\frac{\arg \{p(x, y)\}}{2} \pi\right)\left(\frac{\lambda}{n-1}\right)
$$

where $h$ is the height, $p(x, y)$ is the complex function describing the pupil (Equation 1), $\lambda$ is the emission wavelength and $n$ is the refractive index of the substrate (here, we use fused quartz with $n=1.462$ at $\lambda=510 \mathrm{~nm}$ ). In practice, our realized surface relief is a discrete 8-level approximation of Equation 2 with $\alpha=200$ over an $18 \mathrm{~mm}$ diameter, with an optimal $\lambda \approx 510 \mathrm{~nm}$. A $510 \pm 30 \mathrm{~nm}$ chromatic filter was located immediately after the phase mask. An imaging detector (Andor iXon Ultra 897, electron-multiplying CCD) is located in the imaging plane of the 1:1 relay system.

\section{TARGETING CALIBRATION, IMAGE ACQUISITION AND SIGNAL RECONSTRUCTION}

Precise targeting of the ROIs (neurons) was facilitated by a $3 \mathrm{D}$ calibration of the SLM projection patterns. A calibration phantom was created using a $2 \%$ agarose mixture with a dye solution (yellow highlighter dye) at a 1:1 ratio. Standard projection grids are projected by the SLM at up to $20 \mathrm{z}$-positions through the volume of interest and the resulting fluorescence image was used to calculate the affine transformation that maps the SLM-based illumination pattern to the EM-CCD. A 3rd-order polynomial fit of each element from the axially dependent affine matrix was used to approximate the axial evolution of the transform and stored for later use (Quirin et al., 2013). A pollen grain slide was similarly used to calibrate the axial dependent, affine transformation from the EM-CCD image to the PMT image frame. The slide was translated through the volume range of interest and an EM-CCD image and PMT image were acquired for comparison. An automated fitting routing estimated the axial-dependent affine transform matrix and, as with the SLM to EM-CCD transform, a 3rd-order polynomial fit is made to each element of the matrix. Matrix multiplication of the PMT to EM-CCD and the EM-CCD to SLM transforms yield the coordinates to load onto the SLM for precise targeting. Though it is known that any errors in matching the refractive index of the phantom material to that of the biological sample will result in uncorrected spherical aberration for the structured illumination pattern and that real tissue has inherent inhomogeneity that will further aberrate the illumination beamlets, in practice we find that both of these factors appear negligible when using the above calibration prescription for the axial ranges and samples described in this paper.

Images acquired on the imaging detector in volume projection mode were processed using custom analysis software written in MATLAB (The Mathworks, Natick, MA). Volume projection acquisition mode consists of generating the hologram illumination pattern for the SLM (see Quirin et al., 2013, and references therein for details), setting the EO modulator for appropriate illumination power, opening the shutter and acquiring time-series images that were saved as 16-bit TIFs (image acquisition under the Andor Solis environment). Because the magnified image of the neurons was relatively large $(\sim 200 \mu \mathrm{m}$ per soma) when using an $M=20 \times$ objective compared to the imaging detector pixel size $(16 \mu \mathrm{m})$, pixel binning of $4 \times 4$ was routinely used to improve SNR without any appreciable loss of spatial resolution performance. Using the calibration matrices described above, an experimentally measured PSF (taken from the calibration process) is convolved with the estimated location of each ROI on the CCD to form a basis set of images-one image for each target. Each frame from the time-series stack of images was decomposed into a linear superposition of this basis set using a least-squares fitting, plus one image for background estimation. Formally this was accomplished by creating a matrix of $N$ images,

$$
\boldsymbol{B}=\left[\begin{array}{ccc}
B_{i}(1) & \ldots & B_{N}(1) \\
\ldots & \ldots & \ldots \\
B_{i}(m * n) & \ldots & B_{N}(m * n)
\end{array}\right]
$$

where each column is one $m \times n$ image, lexicographically ordered in a column, representative of the expected pattern from the ROI (known from the deterministic illumination). In principle, these images can be given by simulation or found experimentally. Later acquisition of the experimental image, $\boldsymbol{I}(t)$, at time $t$ (see for example, Figure 1E) can then be characterized by,

$$
\boldsymbol{I}(t)=\boldsymbol{B} \cdot \boldsymbol{W}(t)+\boldsymbol{n}
$$

to describe the image formation where $\boldsymbol{n}$ is additive random noise. The least-squares fitting,

$$
\widehat{\boldsymbol{W}}(t)=\min _{\boldsymbol{W}(t)}\|\boldsymbol{I}(t)-\boldsymbol{B} \cdot \boldsymbol{W}(t)\|^{2}
$$

quickly yields the individual fluorescence from each target, $w_{i}(t)$.

Any systematic movements of the sample would be easily detected as synchronous changes across multiple ROIs, and would not be expected to show the characteristic fast rise and slow decay of calcium transients resulting from action potentials. No such atypical fluorescent changes were detected during our experiments, although controls (gently tapping the microscope stage) showed we can easily detect and distinguish such events.

\section{ANIMAL EXPERIMENTS}

All animal experiments were performed in accordance under approved protocols following the regulations and guidelines of Columbia University's IACUC and ICM. Mice experiments were performed in C57BL/6 mice aged P11 to P60 using $400 \mu \mathrm{m}$ thick coronal slices loaded with FURA-2AM. Zebrafish experiments were performed at ages P6-P8 and were performed in accordance with the regulations and guidelines of Columbia University and the Howard Hughes Medical Institute. The zebrafish sample is held fixed within a bead of $2 \%$ low-melting point agarose to reduce motion artifacts. 


\section{RESULTS}

We first demonstrated the system capabilities using calcium indicators in vitro, in acute mouse hippocampal slices to demonstrate single-cell resolution. Sections of the dentate gyrus were selected for 3D imaging, specifically because this brain structure provides a challenging opportunity with its dense ensemble of cells (Figure 3A). Recent work has reported the presence and importance of functional cell-cluster activity with $2 \mathrm{D}$ imaging in the hippocampus (Muldoon et al., 2013; Ramirez et al., 2013), however these events have never been observed in 3D. We first present an example of the dense, three-dimensional packing of cells in this structure, which was acquired by conventional sequential raster scanning, and was then used to identify the cells for targeting (Figure 3A). Every visually identified cell in the FOV was targeted for illumination by creating and loading the associated hologram pattern on the SLM. In one representative demonstration, data are acquired for 107 such ROIs (an average of
$<11 \mathrm{~mW}$ incident power per spot) and recorded at 55 volume projection images per second (VPPS) with an exposure time of $16.6 \mathrm{~ms}$ per frame (Figures 3B, 4). Individually distinguished fluorescence activity from the dense three-dimensional cluster of cells demonstrates that unique signals from individual neurons can be extracted easily, and with high SNR. Before and after one identified burst, independent activity is observed in each cell body regardless of proximity of the targets in space-demonstrating cellular resolution.

This is perhaps unsurprising after examining the acquired cubic-phase images (Figure 4C). Because SLM-based multisite two-photon excitation preserves the exquisite precision and sensitivity of two photon targeting (Nikolenko et al., 2008), and the cubic phase mask provides reasonable energy compactness (blur is small), and is highly efficient (phase-only modulation), even with dense targeting, simple signal extraction procedures provide high performance. An example of this is shown in Figure 4D,
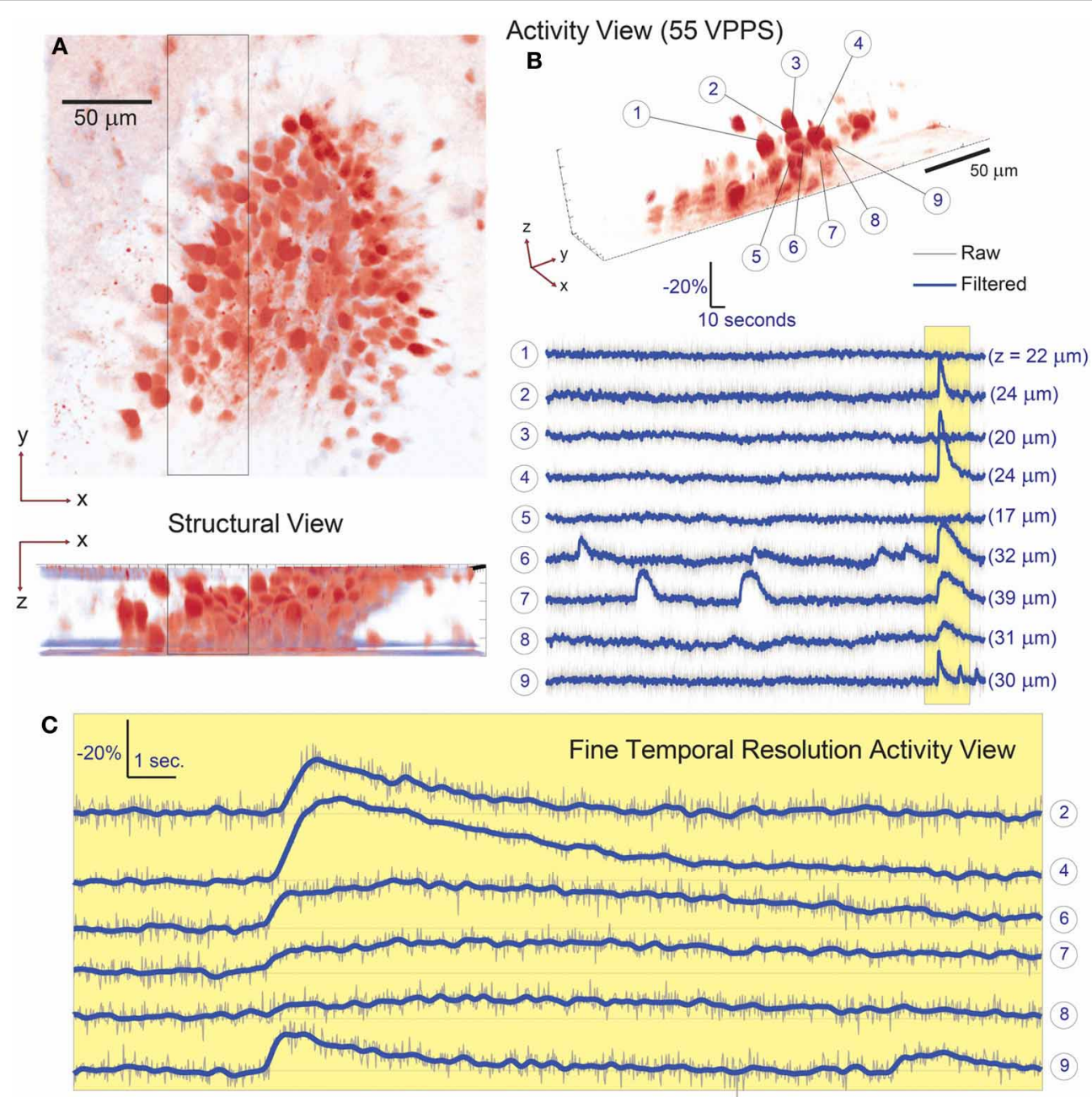

FIGURE 3 | Simultaneous 3D imaging of hippocampal neuronal activity in vitro at 55 volume projections per second. (A) $3 D$ structural data acquired by two-photon raster-scanning image stack. (B) 3D functional imaging with single cell resolution, where 9 of 107 total cells are selected from the 3D volume [boxed region of $(\mathbf{A})]$ and their respective activity is given in (B). Despite simultaneous burst activity in 5 of the neighboring cells, independent calcium transients are detectable. The axial location of each cell is given in parenthesis behind the respective fluorescence trace. (C) A fine-temporal resolution view of the burst activity highlights the variability in both the temporal and amplitude modulation of the calcium transients. 

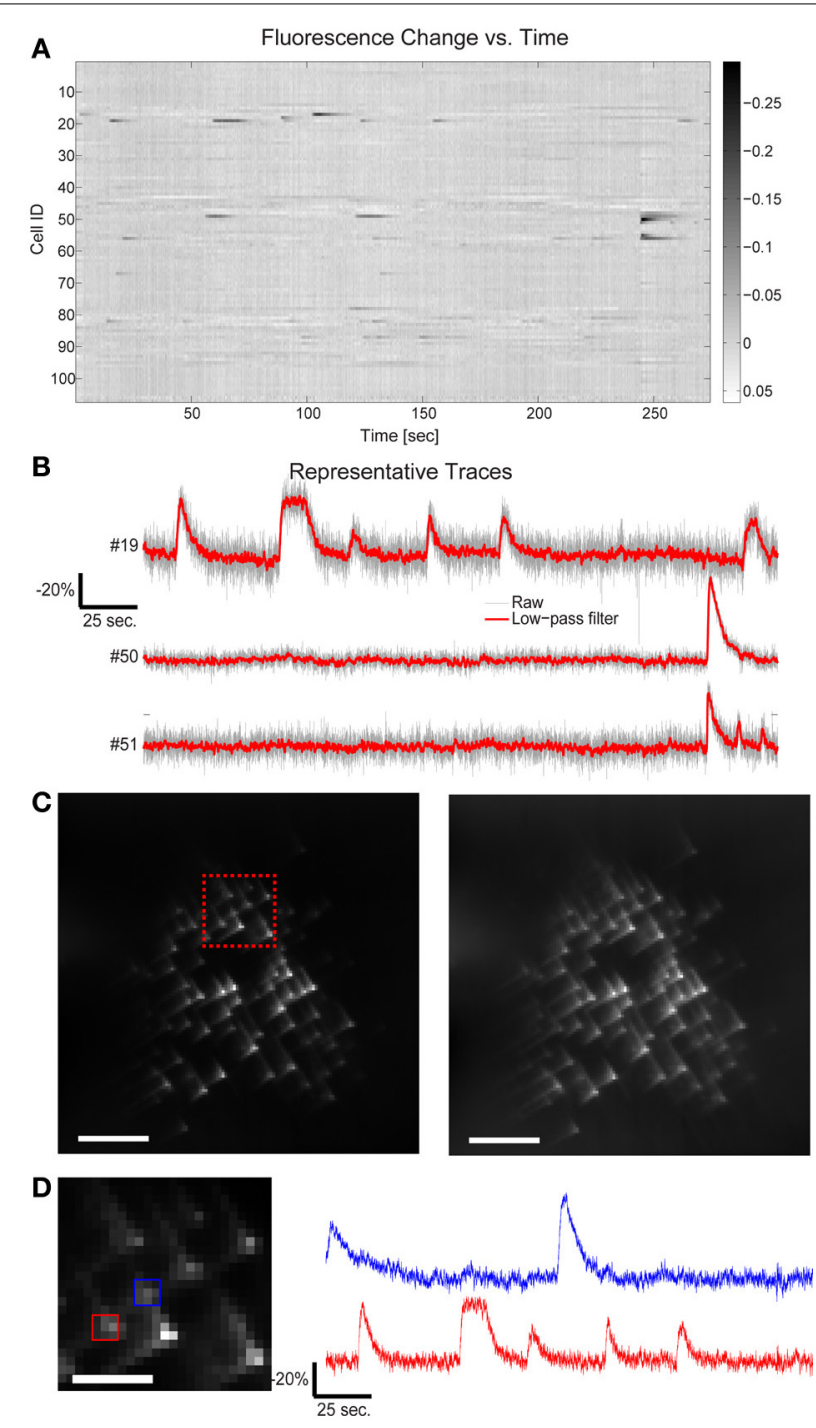

FIGURE 4 | Fluorescence change vs. time for all targets in Figure 3. (A) 109 cells have been targeted by 109 focal spots in the 3D volume and the relative fluorescence change of each targeted location is displayed. Data has been low-pass filtered by a convolution with a Gaussian filter of $\sigma=92 \mathrm{~ms}$. (B) A few representative traces indicate the SNR available by this method. (C) Five second average of the raw acquired images, illustrating both the characteristic cubic-phase aberration and the readily identifiable individual targets. Left image red inset shows area magnified in $4 d$, right frame is the same image, contrast adjusted to highlight dim features. Scale bar is $40 \mu \mathrm{m}$ in both images. (D) Magnified area of image in (C), showing dense targeting, and again illustrating the cubic-phase aberration. Scale bar is $15 \mu \mathrm{m}$. Red and blue boxes indicate ROls used to generate traces shown on the right, using "traditional" DF/F signal extraction, without fitting. The red trace corresponds to cell \#19 in (B), and displays nearly identical absolute modulation and SNR. Note the near absence of crosstalk between the traces, although laterally, the two sources are separated by $\sim 10 \mu \mathrm{m}$. Traces are low-pass filtered as in (A).

which displays a small area of the total image, and shows the DF/F traces extracted with a small ROI over only the bright central lobe of the cubic-phase PSF (not the basis fitting procedure described earlier) for two targeted cells, whose lateral projections are separated by $\sim 10 \mu \mathrm{m}$. These traces show very clean signals, with little to no sign of cross contamination, and show modulation nearly identical to the signal extracted using the least squares fitting procedure (Figure 4B, cell \#19, also shown in Figure 4D). As a practical note, in our laboratory, we use this as a rule-ofthumb for the overall quality and performance of the imaging system. If the central lobes of the images of each target are identifiable as distinct puncta, we find that simple methods for signal recovery, such as our linear fitting procedure, and in many cases, traditional ROI selection, will be sufficient to extract high quality signatures from each target.

A key advantage of this method is that minute differences in the onset timing and calcium dynamics of the $3 \mathrm{D}$ ensemble can now be resolved with high SNR even at these high temporal sampling rates (Figures 3C, 4). In slices from older animals, with less dense labeling, we demonstrated this method at sampling rates up to $125 \mathrm{VPPS}$ ( $<20 \mathrm{~mW}$ incident power per spot and $6.3 \mathrm{~ms}$ exposure times) at single-cell resolution and with high SNR (Figure 5). We have compared the distribution of fluorescent changes and activity patterns collected using the VPI approach to those recorded with a long established imaging method (one photon, laser-illuminated spinning disk confocal, with an EM-CCD, recording at $20 \mathrm{fps}$ ) that were accompanied with cell-attached recordings (Figure 6). The recordings also match prior measurements from our laboratory, where ground truth sensitivity to single action potential was clearly demonstrated using the original planar SLM microscope with Fura-2 loaded cortical slices (Nikolenko et al., 2008). Although this is indirect evidence, we believe this indicates that we should be sensitive and have sufficient SNR in most instances to record single action potentials. Co-firing dentate gyrus cells likely represent ensembles of hippocampal neurons involved in pattern separation (Muldoon et al., 2013; Ramirez et al., 2013). Our technique thus allows for the visualization of these activity patterns with unprecedented spatiotemporal precision.

We also reconstructed the in vivo neuronal activity of the larval zebra fish (P7) with GCaMP5G (Akerboom et al., 2012; Ahrens et al., 2013) to map the temporal record of brain-wide calcium transients at high-speed. Different spatial scales can be accessed with the VPI technique by simply selecting an alternative microscope objective or modulating the strength of the phase aberration present on the wavefront coding element at the pupil (via use of $\alpha$, in Equation 1). As a reference, we show conventional two-photon galvanometric scanning images that contain brain-wide activity patterns where both individual cells and neuropil are recruited and fluoresce (Figures 7A,E) (see also Ahrens et al., 2013; Panier et al., 2013). In recent work, the temporal resolution of such whole-brain activity mapping was limited by the axial scan speed and the camera frame rate, sampling at $0.8 \mathrm{~Hz}$ (Ahrens et al., 2013) or $4 \mathrm{~Hz}$ using selective axial planes (Panier et al., 2013). In contrast, the technique proposed here operates simultaneously across axial planes. Moreover, VPI has no moving parts and therefore exhibits no mechanical acquisition speed limitation, nor the complications that can arise from coupling motion of the system into the sample. For illustration, we targeted 49 randomly distributed cells within a $350 \times 350 \times 150 \mu \mathrm{m}$ volume (on average $<14 \mathrm{~mW}$ incident power per spot). Using these 


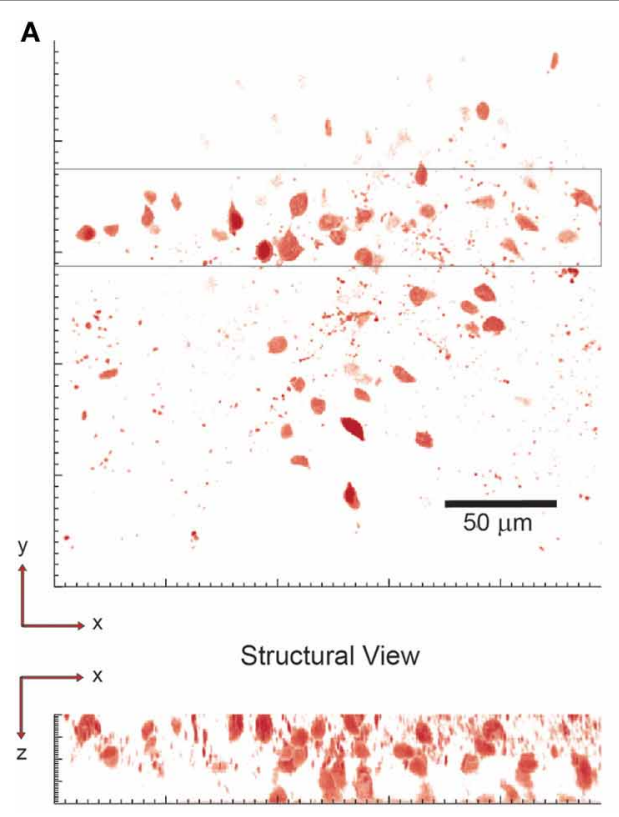

FIGURE 5 | 3D imaging of neuronal activity in dentate gyrus with single-cell resolution at $\mathbf{1 2 5}$ volume projections per second. (A) The location of neuronal cells within the volume of interest is determined by collecting a two-photon raster-scanning image stack and identifying cell bodies. To demonstrate 3D sensing capability with single-cell resolution, 7 neighboring cells are selected from the 3D sub-volume highlighted in

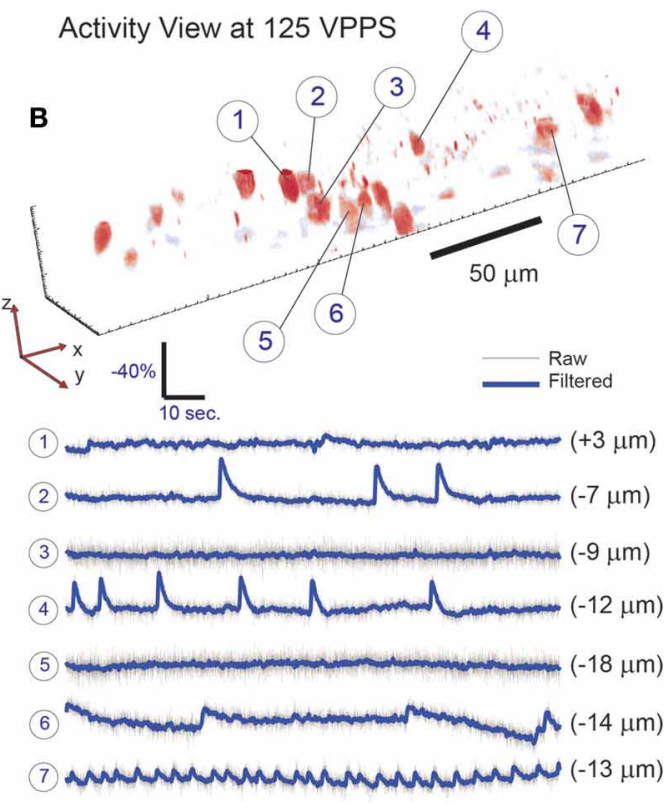

(A) and their respective activity is shown in (B). Note that neighboring cells are seen to have independent activity_confirming single-cell resolution. The axial location of each cell is given in parenthesis behind the respective fluorescence trace. 58 total targets were monitored (out of 61 total labeled cells identified via visual inspection) throughout the tissue volume.
ROIs, a custom hologram was created and loaded to the SLM (red targets, Figure 7B). With the simultaneous multisite twophoton excitation, multiple waves of large scale near-synchronous calcium transients are precisely recorded at 30 VPPS ( $32 \mathrm{~ms}$ exposure times per frame). The monitored active cells in these waves were temporally sorted based on their activity in the first wave, and this same ordering was used to display the activity in the subsequent waves. We observed that ordering is strongly preserved in the subsequent waves, with sub-second precision, despite occurring many minutes later (Figure 7C). It is important to note that this is not an epileptiform or simple activity pattern-the spatiotemporal profile of the activity is dispersed throughout the sample with the cells closest geographically not necessarily having the smallest relative offsets in the onsets of activity (Figure 7D). This is, to our knowledge, the first demonstration of simultaneous three dimensional calcium activity imaging in in vivo zebra fish preparations with single cell precision at a temporal resolution sufficient to resolve the dynamics of neural activity patterns.

\section{DISCUSSION}

Using SLM structured illumination with VPI, we can image the activity of neuronal populations throughout the brain of the larval zebrafish simultaneously. But, although the larval zebra fish is transparent, most living organisms are not. Our method as implemented here still relies on direct optical imaging and is adversely affected in highly scattering environments. This has been a challenge for all optical imaging methods in neuroscience, and the described method is not immune to it. We evaluated the effect of scattering on the PSF used here and believe that this method can be applied up to 2-3 scattering lengths deep providing that a relatively sparse selection of $3 \mathrm{D}$ points is used (Figure 8). However, there are active developments which will extend the absolute depth where this method can be successfully applied. First, ongoing improvements in red shifted indicators and light sources, whose longer wavelengths penetrate more deeply through tissue, could allow for significant and sizeable volumetric imaging with high spatial and temporal resolution. With the development of faster SLMs, or external modulation schemes, temporal coding and multiplexing can be added to augment the selectivity at large imaging depths by imposing an a priori temporal structure on each target, allowing for phase locked detection (Ducros et al., 2013). This could be a critical improvement, as it would allow for imaging neurons whose projections fall within the same pixels of the camera (such as neurons directly on top of one another). Additionally, advances in algorithms for signal extraction that jointly consider the recorded spatial and temporal signatures of each source will improve separation for overlapping and delocalized signals (Pnevmatikakis et al., 2013).

Both the illumination and data acquisition are simultaneous and can target multiple ROIs throughout the volume of tissue allowing for parallel activation and imaging. We have demonstrated high SNR recording of calcium transients at speeds of up to $125 \mathrm{~Hz}$, limited by the camera transfer rate. This frame rate and sensitivity is already well matched to the current generation of GFP based genetically encoded voltage indicators, such as Arclight 
A

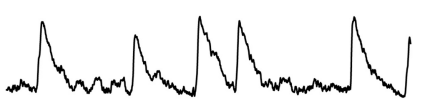

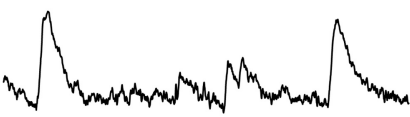

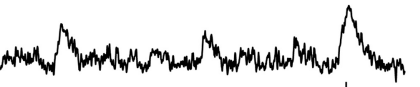
${ }_{20 \mathrm{~s}} 0.1 \mathrm{dF} / \mathrm{F}$
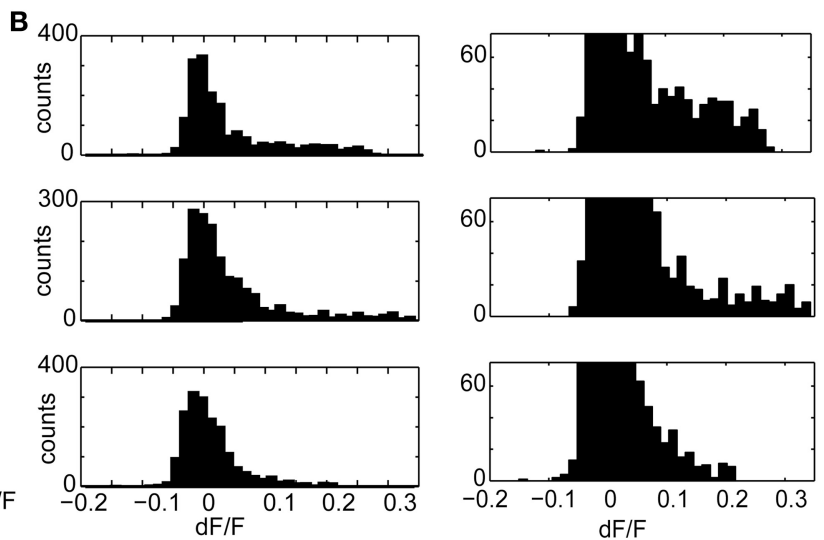

C

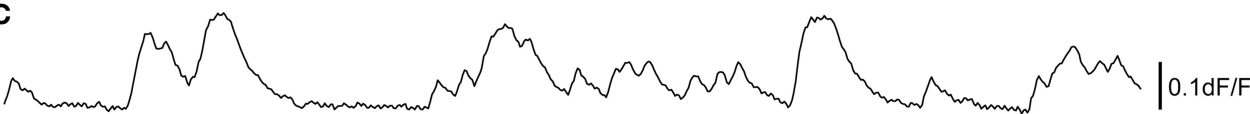

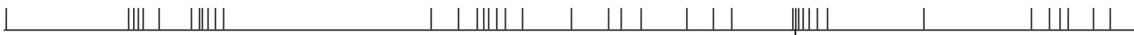

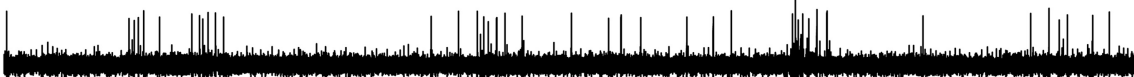

D

E
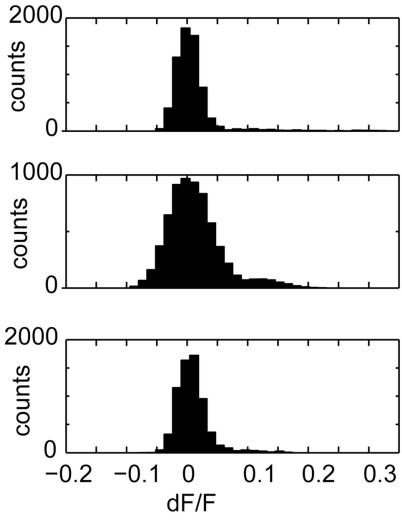

$5 s$
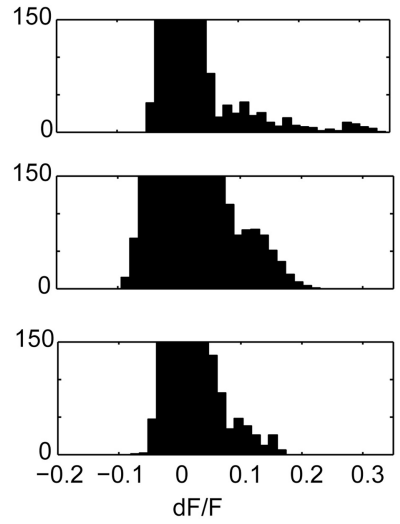

FIGURE 6 | Calcium transients in hippocampal slices display similar amplitudes and kinetics with confocal microscopy and volume projection 3D imaging. (A) Example calcium transients from three representative granule cell ROls, acquired from the dentate gyrus, using a spinning disk confocal microscope. The sample preparation was identical to the brain slice experiments described in the main text. Only a short 2 min segment of the data is shown. Data were acquired at 20 fps. (B) (Left) dF/F distributions for the three ROls shown in (A). On the right is the $y$-axis zoomed histogram, shown to highlight the positively skewed dF/F distributions characteristic of active neuron spiking. (C) $A$ representative experiment showing the ability of Fura-2AM calcium transients to faithfully resolve action potential firing in a cell attached recording. The deconvolved calcium dynamics (above) from a cell recording in cell attached are shown (below) with the filtered $(0.2-4 \mathrm{kHz})$ cell attached recording and threshold crossing spikes. Note the ability of the Fura-2AM to resolve single action potential firing, which was usually accompanied by a $5 \% \mathrm{dF} / \mathrm{F}$. Therefore, in most experiments, single, or at minimum doublet spiking would be detected using calcium imaging.

$(\mathbf{D}, \mathbf{E})$ The same as $(\mathbf{A}, \mathbf{B})$, but with three examples of ROls measured using the $3 \mathrm{D}$ volume projection imaging system described in the manuscript. Data displayed were acquired at 55VPPS, and smoothed with a Gaussian filter to match the sample rate of the confocal images. Note the similar shape calcium transients (D), and dF/F distributions (E). The different size calcium dynamics and the dF/F distributions suggest that single or at minimum doublet spiking can be resolved with the volume projection imaging technique.
(Jin et al., 2012). Advances in the technologies utilized here, such as higher power lasers and increased pixel count SLMs, along with faster cameras will soon accommodate direct imaging of voltage activity in $3 \mathrm{D}$.

In contrast to high speed acousto-optic deflector microscopy techniques, 3D SLM microscopy has the advantage of decoupling the pixel dwell time (time allotted to collect fluorescence signal) from the number of targets. This is an important distinction, and deserves attention. Admittedly, the best fast AOD systems are currently capable of high performance, and set a mark by which all alternative high speed approaches are measured. However fast the performance, we believe they will be difficult to scale significantly beyond their current levels because with very high speed random access approaches, the total collected fluorescence signal per 


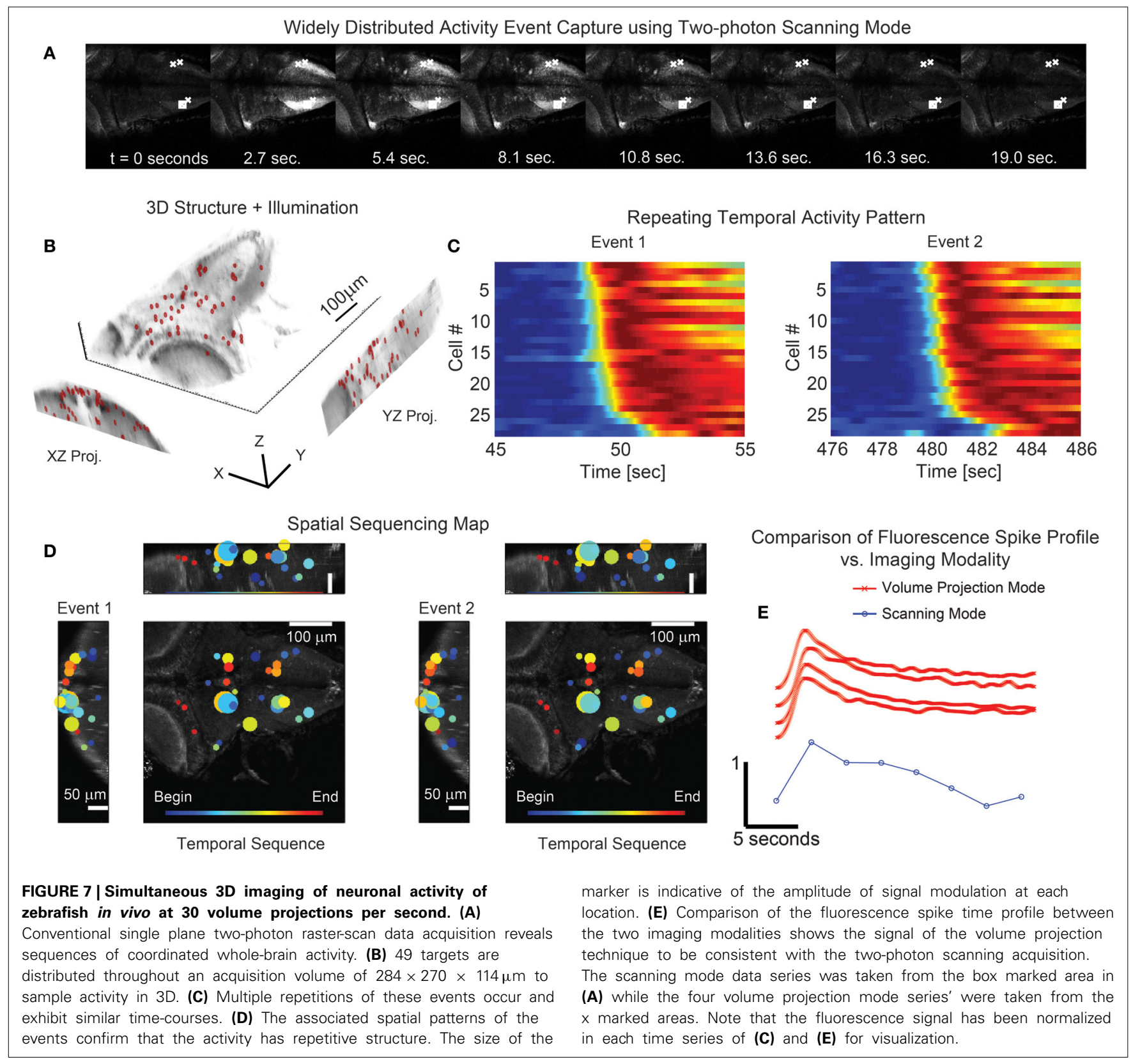

target is limited by the relatively low duty cycle per location and the maximum emission rate of the fluorophore (a physical characteristic of the particular chromophore). Though each target could theoretically receive the full output of the laser, simply increasing the intensity of illumination yields diminishing returns because of fluorophore saturation and continued high intensity excitation will lead to increased photodamage and bleaching, rather than increased signal, along with a loss of spatial resolution (Hopt and Neher, 2001). With SLM microscopy, the issue is different. Each target has very high duty cycle with respect to the sampling rate (true simultaneous multisite illumination), but with lower average instantaneous power-multiplexing the beam results in diminished fluence per target, with a corresponding reduction in total fluorescence per target. In this case, what limits the total number of targets is the overall laser power available, which can be arbitrarily increased until the overall power deposition over the entire FOV exceeds the total acceptable heat load for the sample. We note that with programmable SLMs, one can achieve the optimal synthesis of both methods with simultaneous, multisite random access targeting, offering tremendous flexibility in monitoring activity. Also, while we describe two photon SLM-based multiple beamlet excitation here, any predetermined structured illumination can be used.

In conclusion, we present a technique for fast, simultaneous, two-photon optical data acquisition of neuron activity which is distributed throughout three dimensions. This has been demonstrated in different animal preparations, both in vitro and in vivo, that are relevant for neuroscience. The combination of structured 

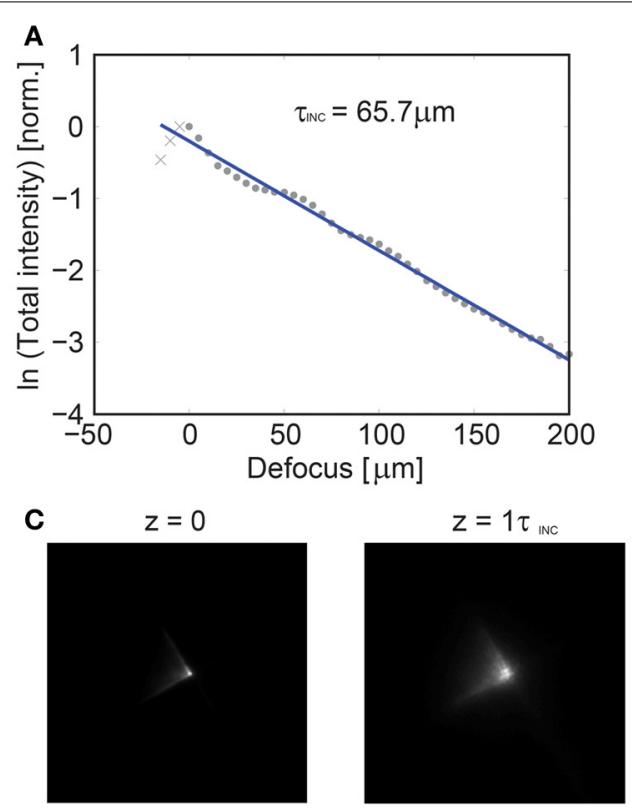

FIGURE 8 | Axial dependence of the imaging PSF vs. depth in scattering tissue. Characterization of the imaging performance of volume projection imaging using GCaMP6s labeled tissue at P31 in white matter tissue. GCaMP6s was chosen to provide near uniform labeling throughout the tissue. (A) A focused spot was translated axially via a mechanical stage and the resulting collected intensity is reported, yielding a mean scattering length of the incident illumination $(\lambda=920 \mathrm{~nm})$ of
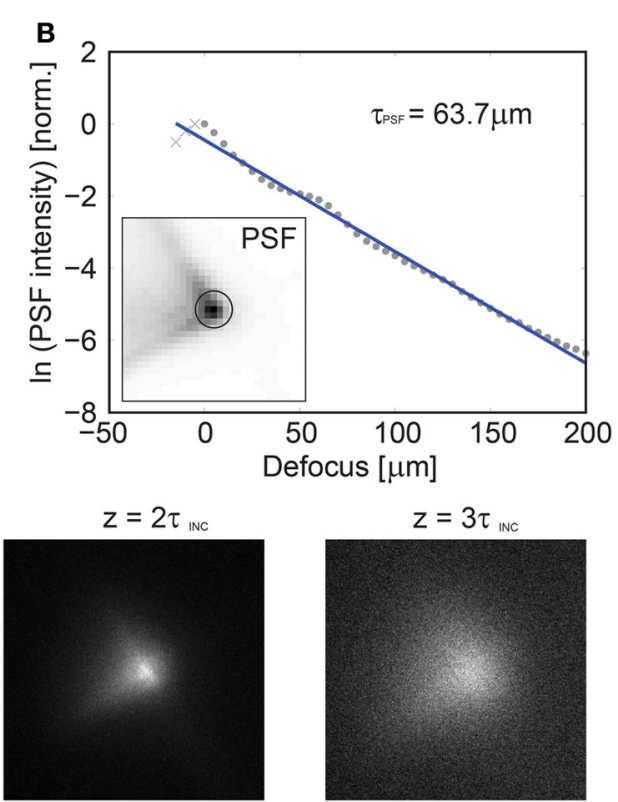

$\tau_{\text {INC }}=65.7 \mu \mathrm{m}$. In an attempt to quantify the loss of the PSF fidelity with increasing scattering, a circular region was defined [centered on the peak intensity, insert (B)] and the relative loss of energy was plotted as a function of depth, yielding $\tau_{P S F}=63.7 \mu \mathrm{m}$ (B). An image of the PSF is given as a function of $\tau_{\mid N C}$ in (C) and the PSF fidelity is observed to degenerate by $\sim 3 \tau_{\mid N C}$, at which point the system performance approaches that of a conventional SLM microscope. illumination with volume projection imaging appears to us a promising platform for future work on brain activity mapping (Alivisatos et al., 2012).

\section{ACKNOWLEDGMENTS}

Sean Quirin, Darcy S. Peterka, Jesse Jackson, and Rafael Yuste are supported by the NIH Director Pioneer Award (DP1EY024503), NIMH (R41MH100895), Keck Foundation and NARSAD. Sean Quirin was additionally supported by the NEI (T32EY013933). This material is based upon work supported by, or in part by, the U.S. Army Research Laboratory and the U.S. Army Research Office under contract number W911NF-12-1-0594. The authors thank Misha Ahrens and Davis Bennett for assistance with the zebrafish preparations.

\section{REFERENCES}

Ahrens, M. B., Orger, M. B., Robson, D. N., Li, J. M., and Keller, P. J. (2013). Wholebrain functional imaging at cellular resolution using light-sheet microscopy. Nat. Methods 10, 413-420. doi: 10.1038/nmeth.2434

Akerboom, J., Chen, T. W., Wardill, T. J., Tian, L., Marvin, J. S., Mutlu, S., et al. (2012). Optimization of a GCaMP calcium indicator for neural activity imaging. J. Neurosci. 32, 13819-13840. doi: 10.1523/JNEUROSCI.2601-12.2012

Alivisatos, A. P., Chun, M., Church, G. M., Greenspan, R. J., Roukes, M. L., and Yuste, R. (2012). The brain activity map project and the challenge of functional connectomics. Neuron 74, 970-974. doi: 10.1016/j.neuron.2012.06.006

Anselmi, F., Ventalon, C., Begue, A., Ogden, D., and Emiliani, V. (2011). Threedimensional imaging and photostimulation by remote-focusing and holographic light patterning. Proc. Natl. Acad. Sci. U.S.A. 108, 19504-19509. doi: 10.1073/pnas. 1109111108

Cathey, W. T., and Dowski, E. R. (2002). New paradigm for imaging systems. Appl. Opt. 41, 6080-6092. doi: 10.1364/AO.41.006080
Chen, T. W., Wardill, T. J., Sun, Y., Pulver, S. R., Renninger, S. L., Baohan, A., et al. (2013). Ultrasensitive fluorescent proteins for imaging neural activity. Nature 499, 295-300. doi: 10.1038/nature12354

Cheng, A., Goncalves, J. T., Golshani, P., Arisaka, K., and Portera-Cailliau, C. (2011). Simultaneous two-photon calcium imaging at different depths with spatiotemporal multiplexing. Nat. Methods 8, 139-142. doi: 10.1038/nmeth.1552

Dowski, E. R., and Cathey, W. T. (1995). Extended depth of field through wavefront coding. Appl. Opt. 34, 1859-1866. doi: 10.1364/AO.34.001859

Ducros, M., Houssen, Y. G., Bradley, J., Sars, V. D., and Charpak, S. (2013). Encoded multisite two-photon microscopy. Proc. Natl. Acad. Sci. U.S.A. 110, 13138-13143. doi: 10.1073/pnas.1307818110

Gobel, W., Kampa, B. M., and Helmchen, F. (2007). Imaging cellular network dynamics in three dimensions using fast 3D laser scanning. Nat. Methods 4, 73-79. doi: 10.1038/nmeth989

Grynkiewicz, G., Poenie, M., and Tsien, R. Y. (1985). A new generation of Ca2+ indicators with greatly improved fluorescence properties. J. Biol. Chem. 260, 3440-3450.

Hopt, A., and Neher, E. (2001). Highly nonlinear photodamage in two-photon fluorescence microscopy. Biophys. J. 80, 2029-2036. doi: 10.1016/S00063495(01)76173-5

Horton, N. G., Wang, K., Kobat, D., Clark, C., Wise, F., Schaffer, C., et al. (2013). In vivo three-photon microscopy of subcortical structures of an intact mouse brain. Nat. Photonics 7, 205-209. doi: 10.1038/nphoton.2012.336

Jin, L., Han, Z., Platisa, J., Wooltorton, J. R., Cohen, L. B., and Pieribone, V. A. (2012). Single action potentials and subthreshold electrical events imaged in neurons with a fluorescent protein voltage probe. Neuron 75, 779-785. doi: 10.1016/j.neuron.2012.06.040

Katona, G., Szalay, G., Maak, P., Kaszas, A., Veress, M., Hillier, D., et al. (2012). Fast two-photon in vivo imaging with three-dimensional randomaccess scanning in large tissue volumes. Nat. Methods 9, 201-208. doi: 10.1038/ nmeth. 1851

Kralj, J., Douglass, A. D., Hochbaum, D. R., Maclaurin, D., and Cohen, A. E. (2012). Optical recording of action potentials in mammalian neurons using a microbial rhodopsin. Nat. Methods 9, 90-95. doi: 10.1038/nmeth.1782 
Muldoon, S. F., Soltesz, I., and Cossart, R. (2013). Spatially clustered neuronal assemblies comprise the microstructure of synchrony in chronically epileptic networks. Proc. Natl. Acad. Sci. U.S.A. 110, 3567-3572. doi: $10.1073 /$ pnas. 1216958110

Nikolenko, V., Watson, B. O., Araya, R., Woodruff, A., Peterka, D. S., and Yuste, R. (2008). SLM Microscopy: scanless two-photon imaging and photostimulation using spatial light modulators. Front. Neural Circuits 2:5. doi: 10.3389/neuro.04.005.2008

Packer, A. M., Peterka, D. S., Hirtz, J. J., Prakash, R., Deisseroth, K., and Yuste, R. (2012). Two-photon optogenetics of dendritic spines and neural circuits. Nat. Methods 9, 1202-1205. doi: 10.1038/nmeth.2249

Panier, T., Romano, S. A., Olive, R., Pietri, T., Sumbre, G., Candelier, R., et al. (2013). Fast functional imaging of multiple brain regions in intact zebrafish larvae using selective plane illumination microscopy. Front. Neural Circuits 9:65 doi: 10.3389/fncir.2013.00065

Papagiakoumou, E., Anselmi, F., Begue, A., de Sars, V., Gluckstad, J., Isacoff, E. Y., et al. (2010). Scanless two-photon excitation of channelrhodopsin-2. Nat. Methods 7, 848-854 doi: 10.1038/nmeth.1505

Pnevmatikakis, E. A., Machado, T. A., Grosenick, L., Poole, B., Vogelstein, J. T., and Paninski, L. (2013). Rank-penalized Nonnegative Spatiotemporal Deconvolution and Demixing of Calcium Imaging Data. Available online at: http://www.stat.columbia.edu/ liam/research/abstracts/cosyne13/eftychios.pdf

Quirin, S., Peterka, D. S., and Yuste, R. (2013). Instantaneous three-dimensional sensing using spatial light modulator illumination with extended depth of field imaging. Opt. Express 21, 16007-16021. doi: 10.1364/OE.21.016007
Ramirez, S., Liu, X., Lin, P.-A., Suh, J., Pignatelli, M., Redondo, R. L., et al. (2013). Creating a false memory in the Hippocampus. Science 341, 387-391. doi: 10.1126/science. 1239073

Schrödel, T., Prevedel, R., Aumayr, K., Zimmer, M., and Vaziri, A. (2013). Brainwide $3 \mathrm{D}$ imaging of neuronal activity in Caenorhabditis elegans with sculpted light. Nat. Methods 10, 1013-1020. doi: 10.1038/nmeth.2637

Yuste, R., and Katz, L. C. (1991). Control of postsynaptic calcium influx in developing neocortex by excitatory and inhibitory neurotransmitters. Neuron 6, 333-344. doi: 10.1016/0896-6273(91)90243-S

Conflict of Interest Statement: The authors declare that the research was conducted in the absence of any commercial or financial relationships that could be construed as a potential conflict of interest.

Received: 03 January 2014; paper pending published: 31 January 2014; accepted: 10 March 2014; published online: 03 April 2014.

Citation: Quirin S, Jackson J, Peterka DS and Yuste R (2014) Simultaneous imaging of neural activity in three dimensions. Front. Neural Circuits 8:29. doi: 10.3389/fncir. 2014.00029

This article was submitted to the journal Frontiers in Neural Circuits.

Copyright (c) 2014 Quirin, Jackson, Peterka and Yuste. This is an open-access article distributed under the terms of the Creative Commons Attribution License (CC BY). The use, distribution or reproduction in other forums is permitted, provided the original author(s) or licensor are credited and that the original publication in this journal is cited, in accordance with accepted academic practice. No use, distribution or reproduction is permitted which does not comply with these terms. 